\title{
PHYSICS OF THE RHYTHMIC APPLAUSE
}

\author{
Z. Néda, E. Ravasz \\ Babeş-Bolyai University, Dept. of Theoretical Physics \\ str. Kogălniceanu nr.1, RO-3400, Cluj-Napoca, Romania \\ T. Vicsek \\ Eötvös-Loránd University, Dept. of Biological Physics \\ Budapest, Hungary \\ Y. Brechet \\ LTPCM-ENSEEG/INPG, Domaine Universitaire de Grenoble \\ B.P. 75, 38402 Saint Martin d'Heres, Cedex, France \\ A.L. Barabási \\ University of Notre Dame, Department of Physics \\ IN 46556, U.S.A \\ (Last revised: 7 February 2000)
}

\begin{abstract}
We report on a series of measurements aimed to characterize the development and the dynamics of the rhythmic applause in concert halls. Our results demonstrate that while this process shares many characteristics of other systems that are known to synchronize, it also has features that are unexpected and unaccounted for in many other systems. In particular, we find that the mechanism lying at the heart of the synchronization process is the period doubling of the clapping rhythm. The characteristic interplay between synchronized and unsynchronized regimes during the applause is the result of a frustration in the systems. All results are understandable in the framework of the Kuramoto model.

PACS numbers: 89.90.+n, 05.45.Xt, 05.65.+b
\end{abstract}

\section{INTRODUCTION}

Everybody experienced after a good performance the audience showing it's appreciation with a thunderous synchronized clapping. This is suddenly arising from the initially incoherent clapping of individuals and might disappear and reappear again several times during the applause. This synchronization process in the concert hall offers a wonderful example of social self-organization, and it is believed to be the human scale example of the synchronization processes observed in numerous systems in nature. As detailed in [1] spontaneous synchronization occurs in many biological and sociological systems: fireflies in Southeast Asia synchronize their flashes; crickets synchronize their chirping; neural cells of the brain synchronize voltage fluctuations; pacemaker cells in the heart synchronize their fire; womens living together for long times find their menstrual cycle synchronized.

The accepted way of modeling the synchronization process is by considering coupled non-linear oscillators. Understanding the synchronization process of coupled oscillators is an old problem in physics, mathematics and theoretical biology [2]. It is believed that the problem goes back to Huygens, who realized first the synchronization of pendulum clocks hanging together on a wall. While the synchronization of identical coupled oscillators interacting with phase-minimizing interactions is obvious, the problem is non-trivial if one considers a population of non-identical oscillators. Depending on the strength and type of the interaction, and the dispersion of the oscillators frequencies, synchronization might, or might not appear. For biological populations it is crucial to consider non-identical internal frequencies, since individuals are not rigorously equivalent. In this way in order to understand synchronization in biological or sociological systems we have to consider the more complex problem of coupled non-identical oscillators.

For a theoretical study of coupled oscillator models many interaction types were studied. Considering mathematically coupled maps [3] the problem became interesting mostly from the view-point of dynamical systems and chaos. Since most of the coupling processes in nature are through pulse-like interactions (firing of neurons, flashes of fireflies, clapping etc.) a very realistic pulselike coupling is considered in the integrate and fire type models [4.5. In these models the firing of an oscillator results in a phase-jump of all other oscillators. Because the phase-evolution of each oscillator is considered non-linear in time, under very general conditions the jumping process leads to synchronization. Interactions continuously present in time and thus memory effects are considered in the models introduced by Winfree [6] and Kuramoto [7. In these later models we have phase-difference minimizing interactions between globally coupled rotators. For 
a suitable chosen interaction form, the problem becomes exactly solvable and leads to very interesting results.

Although at a first choice the integrate and fire type models look the most promising to understand the synchronization and dynamics of the rhythmic applause, we will argue in favor of the phase-coupled Kuramoto model. According to our interpretation of the phenomenon in the appearance of rhythmic applause memory effects are crucial. By considering the pulse-like interaction of integrate and fire models this important ingredient is totally neglected. In contrast, the continuous phase-coupling in the Kuramoto model offers a first approach to deal with the relevant memory effects.

The purpose of our present paper is to reveal some interesting and new peculiarities of the rhythmic applause, and to understand the observed phenomenon in the framework of the well-known Kuramoto model. Of course, in the context of the rhythmic applause the Kuramoto model should be viewed as an abstract theoretical representation of the situation, containing the minimum number of assumptions already allowing the kind of synchronization exhibited by the spectators. We mention that the main results of our study was briefly discussed in a very short recent communication [8]. Here we will consider a much more detailed and argumented presentation.

\section{THE KURAMOTO MODEL}

In order to construct a simple physical model for synchronization one can take a population of non-identical rotators globally coupled through phase-difference minimizing interactions. Synchronization in this system is not obvious at all and triggered the interesting studies of Winfree [6]. He found that mutual synchronization is possible or not depending on the relation between the oscillators frequency distribution width (dispersion) and the strength of coupling. Later, Kuramoto proposed, and together with Nishikava exactly solved [7] an elegant reformulation of Winfree's model.

In the Kuramoto model we have $N$ rotators each of them described by it's $\phi_{K}$ phase. The rotators have a $g(\omega)$ distribution of their $\omega_{K}$ natural frequencies. Every rotator interacts with all the other ones via phasedifference minimizing terms:

$$
W_{K}^{i n t}=\frac{K}{N} \sum_{j=1}^{N} \sin \left(\phi_{j}-\phi_{K}\right)
$$

The $N$ coupled differential equations describing the overdamped oscillators dynamics are:

$$
\frac{d \phi_{K}}{d t}=\omega_{K}+\frac{K}{N} \sum_{j=1}^{N} \sin \left(\phi_{j}-\phi_{k}\right)
$$

Mathematically the synchronization level will be characterized by an order parameter, $q$, defined at any timemoment as:

$$
q=\left|\frac{1}{N} \sum_{j=1}^{N} e^{i \phi_{K}}\right|
$$

The maximal possible value $q=1$ corresponds to total synchronization, the case $0<q<1$ to partial synchronization, while for $q=0$ there is no synchronization at all in the system.

In the $N \rightarrow \infty$ thermodynamic limit of the equilibrium dynamics $(t \rightarrow \infty$, so initial transient effects are lost) Kuramoto and Nishikava proved the existence of a $K_{c}$ critical coupling. For a Gaussian distribution of the oscillators natural frequencies, characterized by a $D$ dispersion they got [7]:

$$
K_{c}=\sqrt{\frac{2}{\pi^{3}}} D
$$

For $K \leq K_{c}$ the only possible solution gives $q=0$ (no synchronization) while for $K>K_{c}$ a stable solution with $q \neq 0$ appears. Thus, the main result is that for a population of globally coupled non-identical oscillators a partial synchronization of the phases is possible whenever the interaction among oscillators exceeds a critical value. By adopting the Kuramoto model for biological populations the observed synchronization phenomenon are conceptually understood.

\section{RHYTHMIC APPLAUSE VERSUS THE KURAMOTO MODEL: OPEN QUESTIONS}

As we argued in the introduction it is convenient to use the Kuramoto model in order to describe the dynamics of the rhythmic applause. If the Kuramoto model is applicable to the phenomenon there could be two cases: i.) the average coupling between the spectators is smaller than the critical one and no synchronization appears, ii.) for larger coupling than the critical one synchronization would gradually evolve. The critical coupling (as discussed in section 2) is governed by the dispersion of the spectators natural clapping frequencies (4).

Studying carefully the rhythmic applause one might raise immediately many questions which are not obviously answered within a simple application of the $\mathrm{Ku}$ ramoto model:

- Usually at the beginning of the applause there is a long "waiting" time without any synchronization, and with no increase in the order parameter. The partial synchronization is evolving after that suddenly and achieves its maximal value in a short time. This should be not the case if we are in the $K>K_{c}$ limit. One would expect in this limit a continuous increase in the order parameter right from the beginning of the applause. 
- Why synchronization already achieved is lost after a time, and why might reappear again? Loosing synchronization should not happen in the $K>K_{c}$ limit.

In order to understand more deeply the phenomenon and to answer the questions which are not obvious within the framework of the Kuramoto model we first considered an experimental study.

\section{EXPERIMENTAL STUDY}

Two main experiments were considered:

I. The applause after many good theater and opera performances (in Romania and Hungary) was recorded, digitized and analyzed in several aspects. Recordings both by a microphone hanging form the ceiling of the concert hall and in the neighborhood of randomly selected individuals were considered.

II. Well-controlled clapping experiments were carried out on a group of 73 high-school students. We also investigated the clapping frequencies of one individual during a one week period.

\section{A. Experimental method}

I. By digitizing the signal (Fig. 1a) we obtained a recording of fluctuating voltage with both positive and negative values. The zero level (as the mean-signal level) was determined and the square of the signal relative to this level was computed (Fig. 1b). This signal would roughly correspond to the noise intensity variation, but due to the short sampling time it is definitely not the one that our human perception can follow. The average on a relatively short-time period $(\approx 0.2 s)$ was considered and we got the signal (Fig. 1c) which describes well the useful noise-intensity variations during the applause. Already at a first-look one can easily detect the part where the rhythmic applause appears and the periodic noise intensity variation during synchronized clapping.

This short-time averaged signal was analyzed in several aspects:

1. A long-time averaged noise-intensity was computed by averaging the signal on a time interval of approximately $3 s$.

2. An experimentally computable order-parameter, $q_{\text {exp }}$ was calculated. This order-parameter was defined in a very analogous way with the $q$ order parameter (3) in the Kuramoto model. At each timestep $q_{\text {exp }}$ is calculated as the maximum of the normalized correlation between the $s(t)$ signal and a harmonic function

$$
q_{\exp }(t)=\max _{\{T, \phi\}}\left\{\frac{\int_{t-T}^{t+T} s(t) \sin (2 \pi / T+\phi) d t}{\int_{t-T}^{t+T} s(t) d t}\right\}
$$

(in the above formula the values of $\phi$ should span all possible initial phases between 0 and $2 \pi$, and $T$ should vary between two reasonably chosen limiting values, $T_{\min }$ and $T_{\max }$.) Taking $T_{\min }=0.1 \mathrm{~s}$ and $T_{\max }=5 s q_{\text {exp }}$ was numerically computed.

3. Finally, for recordings taken in the neighborhood of one spectator one can observe that the short-time averaged noise intensity curves have some evident local maximums corresponding to the clapping of the individual. In this case we computed the timeinterval between the clearly distinguishable groups of maxima as a function of time. This will characterize the clapping frequency of the chosen spectator.

II. In our well-controlled clapping experiments we studied the distribution function for the natural clapping frequency of non-interacting students. Separated by the rest of the group, students were first encouraged to clap in the manner they would right after a good performance (mode I clapping). Second, they were asked to clap in the manner they would do it during synchronized clapping (mode II clapping). On one student we also performed investigations on the both two ways of clapping, sampling 100 times during a one-week period.

\section{B. Experimental Results}

I. A characteristic part of the global applause where the synchronization is formed and destroyed is presented as a function of time on Fig. 2a. On Fig. 2b. we present simultaneously the recording made in the vicinity of one spectator. The order parameter variation computed by equation 5 from the global signal is plotted on Fig. 2c. On Fig. 2d we show the variation of the long time moving average (window size: $3 \mathrm{~s}$ ) for the global noise intensity. Finally, on Fig. 2e we present the computed clapping period as a function of time for the chosen individual. One can notice immediately that during the rhythmic applause (central part) the long-time averaged noise intensity has a clear minimum, the order parameter has a maximum (as expected) and the clapping period of an individual presents also a maximum. These results are stable and qualitatively consistent with all the 47 recordings we have studied.

II. For the normalized distribution of the natural clapping frequencies of the group of 73 students the results are given on Fig. 3. With continuous line we plotted the distribution obtained for mode I clapping and with dashed line the results for mode II clapping. Both distributions are roughly Gaussian. The maximum of the first 
distribution is located at a higher frequency (approximatively two times higher) and the peak is wider than the one for mode II clapping. Calculating the dispersion for the two distributions, we got both the dispersion $(D)$ and the relative dispersion $\left(D_{r}=D / \bar{\omega}\right)$ for mode II. clapping smaller:

$$
\begin{aligned}
& \frac{D_{I}}{D_{I I}} \approx 2.5 \\
& \frac{D_{I}^{r}}{D_{I I}^{r}} \approx 1.3
\end{aligned}
$$

Computing the normalized distribution for the ratio of mode I to mode II clapping for each individual we got also a one-peek distribution centered around the value of 2 (Fig. 4).

The normalized distribution of mode I and mode II clapping obtained from 100 measurements on one individual (Fig. 5) shows a similar behavior with the one presented in Fig. 4. We plotted with continuous line the normalized distribution obtained for mode I clapping and with dashed line the one for mode II clapping. The distribution of possible natural frequencies during mode II clapping is again sharper than the one for mode I clapping. Again, the location of the maxima for the distribution of mode I clapping is roughly double than the one for mode II clapping.

\section{DISCUSSION}

From the controlled clapping experiments (Figs. 3-5) we conclude that spectators can clap with two very distinct clapping modes. During rhythmic applause they shift from their original high frequency mode I clapping to the slow frequency, mode II clapping. The above picture is totally supported by the clapping rhythm of one individual during the rhythmic applause (Fig. 2e). During this period-doubling process the dispersion (width) of the natural clapping frequencies distribution is decreased roughly to half. This is a very important step in understanding the phenomenon from the viewpoint of the Kuramoto model. From the Kuramoto model we learned that the critical coupling, $K_{c}$, for synchronization is directly proportional with the dispersion of the frequency distribution of the oscillators (4). When the dispersion is decreased the value of the critical coupling decreases too. When the clapping starts the spectators clap very enthusiastically and with high frequencies (mode I clapping). The frequency distribution is wide and the value of the critical coupling is large. The coupling between the spectators is lower than the critical value and synchronization is not possible. The spectators inconstiently realize this (or already inconstiently know the "game" of the rhythmic applause) and by roughly doubling their clapping periods they shift to mode II clapping. By this the dispersion of the natural frequencies of the spectators reduces to half and so does also the value of the
$K_{c}$ critical coupling. The coupling among spectators can become in this way larger than $K_{c}$ and partial synchronization (rhythmic applause) appears.

It is clear now why synchronization appears, but the question remains: why after a time this synchronization is lost? The answer is immediate by examining the long-time averaged noise intensity curve (Fig. 2c). One can realize that after synchronization occurs the average noise decreases and attains its minimal value. Enthusiastic spectators are not satisfied with this and try to increase the average noise intensity level. This is possible mainly by speeding up the rhythm, because the intensity of one clap cannot be increased over a given level any more simply by hitting stronger. Speeding up the rhythm increases the natural frequency distribution's dispersion and consequently increases by this the value of $K_{c}$. The coupling among spectators becomes again smaller than the critical one and synchronization is lost.

The above scenario is fully supported by the analyzed signal in Fig. 2. We learn from here that during the rhythmic applause the individuals in the crowd double their clapping period (Fig. 2e), and we observe how by this the order-parameter characterizing synchronization is increased (Fig. 2d). When the order-parameter is maximum the average noise intensity (Fig. 2c) is minimum and spectators will increase this by increasing the frequency of clapping shifting again to mode II clapping.

The clapping after a good performance is frustrated in some sense. When maximal synchronization is achieved the average noise intensity is minimum due to the slow frequency of mode II clapping. By increasing the clapping frequency and shifting to mode I clapping the value of critical coupling is increased and synchronization is lost. The spectators cannot achieve both maximal noise intensity and good synchronization within the same clapping mode, and this makes the system frustrated.

A very simple computer simulation exercise on the $\mathrm{Ku}-$ ramoto model can visualize all the above results (Fig. 6). Considering an ensemble of $N=70$ globally coupled rotators with a Gaussian distribution of their natural frequencies $\left(\bar{\omega}=2 \pi s^{-1}, D=2 \pi / 6.9 s^{-1}\right.$ and $\left.K=0.8 s^{-1}\right)$ we follow up their dynamics. We associate a very small time length $\tau=0.01 s$ and $I_{0}=\omega / \bar{\omega}$ intensity noise for each event when a rotator passes through an integer multiple of phase $2 \pi$. The global noise is obtained by simply adding all noises generated by the individual rotators. In the given setup we have $K<K_{c}$, and thus no synchronization should appear. However, at $t_{1}=21 \mathrm{~s}$ time moment we double the natural oscillation period of each oscillators, and as observable in Fig. 6a synchronization gradually evolves. This leads to an increase in the orderparameter (Fig. 6b, computed now as in experiments after equation 5), and a decrease in the long-time averaged noise intensity (Fig. 6c). Beginning with $t_{2}=35 \mathrm{~s}$ we begin to linearly increase the rotators natural frequencies back to their original values. As observable, this will decrease again the order parameter and increase the averaged noise intensity. This computer exercise approxi- 
mates well the experimental results from Fig. 2, and gives confidence in our analytical statements.

Some interesting comments can be made at this point.

1. In many societies the phenomenon of rhythmic applause is unknown. It seems that the "game" of the rhythmic applause has to be learned by each community. Spectators once inconstiently recognizing that lowering the clapping frequency allows synchronization and getting familiar to the phenomenon they will keep alive this habit.

2. Usually, in huge open air concerts no clear rhythmic applause forms even after outstanding performances. This is mainly due to the small and non-global coupling existing in the system (the applause of far-away spectators is totally undetectable). Even by reducing to half the natural clapping frequencies dispersion it is still not possible to achieve a coupling larger than the critical one.

3. In communist times it was a common habit to applaud by rhythmic applause the "great" leader speech. During this rhythmic applause the synchronization was almost never lost. This is a very nice evidence of the fact that spectators were not enthusiastic enough and were satisfied with the obtained global noise intensity level, having no desire to increase it. Frustration was not present in this system.

\section{CONCLUSIONS}

By recognizing two characteristic clapping modes of the individuals during the applause and by applying the results of the Kuramoto model the dynamics of the rhythmic applause is qualitatively understood. The rhythmic applause is formed by lowering (roughly to half) the natural clapping frequency of each individual, and thus by lowering the width of the clapping frequency distribution of the audience. The rhythmic applause is lost and might reaper again due to two main desires of the spectators which can not be both fulfilled at the same time: optimal synchronization and maximal applause intensity. The system is frustrated in this sense. When maximal synchronization is achieved the average noise intensity is minimum due to the slow clapping frequency. Increasing the average noise intensity by increasing the clapping frequency synchronization gets lost. The amazing interplay between unsynchronized and synchronized clapping is a consequence of this frustration.
[1] S.H. Strogatz and I. Stewart, Scientific American, December 1993, p. 102; S.H. Strogatz, Lecture Notes in Biomathematics 100 (1993)

[2] L. Glass and M. Mackey, From Clocks to chaos: The Rhythms of Life (Princeton University Press, Princeton, N.J, 1988)

[3] W.Just, Phys. Rep. 290, 101 (1997); Y. Jiang, Phys. Rev. E 56, 2672 (1997); M.G. Rosenblum, A.S. Pikovsky and J. Kurths, Phys. Rev. Lett. 76, 1804 (1996)

[4] R. Mirollo and S. Strogatz, SIAM J. Appl. Math. 50, 1645 (1990)

[5] S. Bottani, Phys.Rev. E 54, 2334 (1997)

[6] A.T. Winfree; J. Theor. Biol. 16, 15 (1967)

[7] Y. Kuramoto and I. Nishikava; J. Stat. Phys. 49, 569 (1987)

[8] Z. Néda, E. Ravasz, Y. Brechet, T. Vicsek and A.L. Barabási; Nature (in press) 


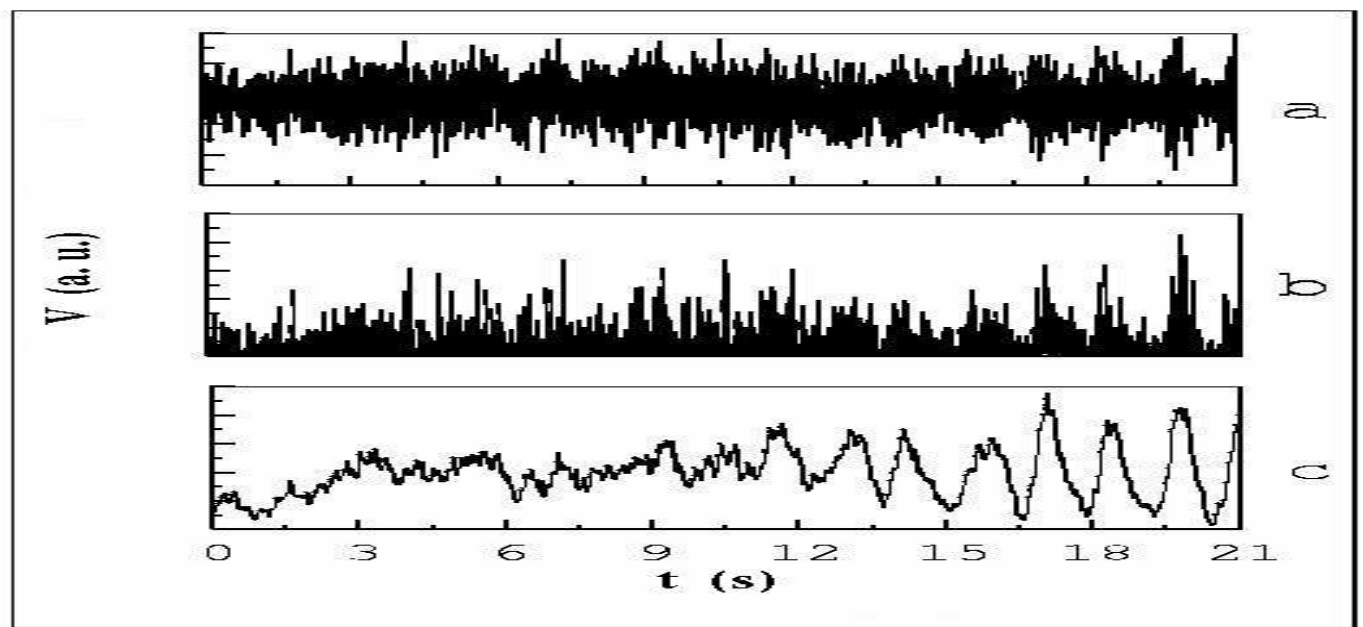

FIG. 1. Digitized global signal, voltage as a function of time $(a)$. The processed signals: $(b)$ and $(c)$. On figure $b$ we have the square of the signal from figure $a$, relative to the calculated average voltage level. On figure $c$ we present the short-time moving average (window size: $0.2 s$ ) of the signal from figure $b$.

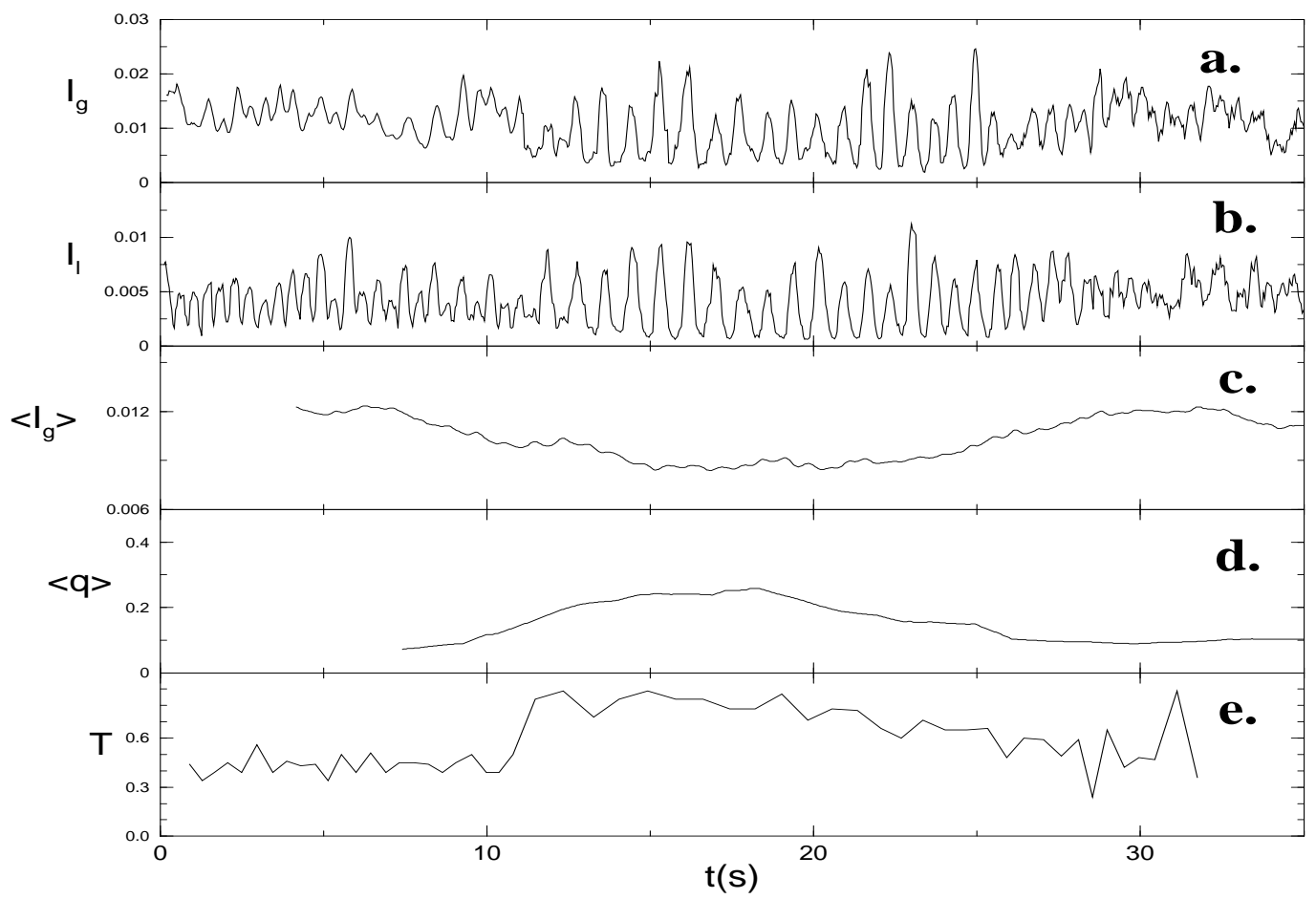

FIG. 2. Short-time averaged global $(a)$ and local $(b)$ signal as a function of time. The computed long-time averaged signal (window size: $3 s$ ) is presented on figure $c$, the experimental order parameter on figure $d$, and the clapping period of the chosen individual on figure $e$. 


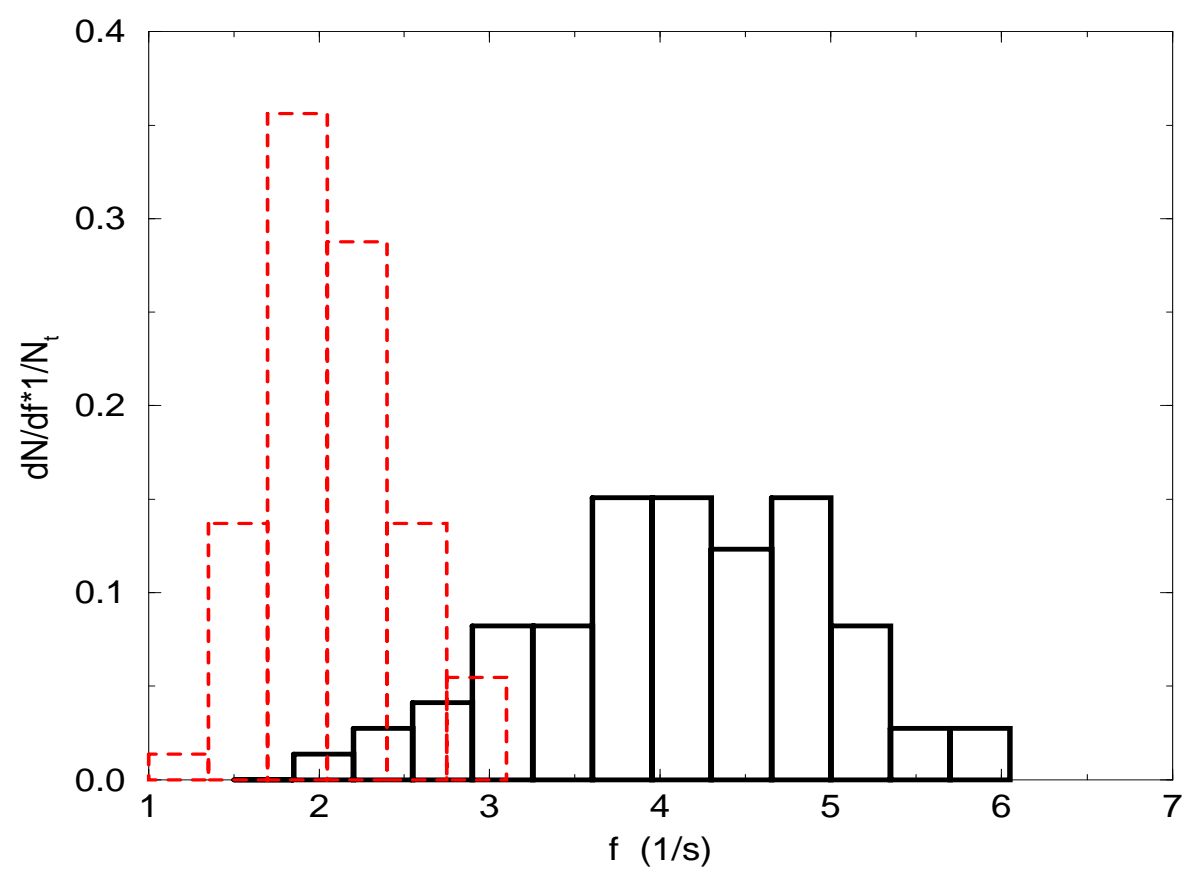

FIG. 3. Normalized distribution functions for mode I (continuous line) and mode II (dashed line) clapping frequencies on a sampling of 73 high-school students.

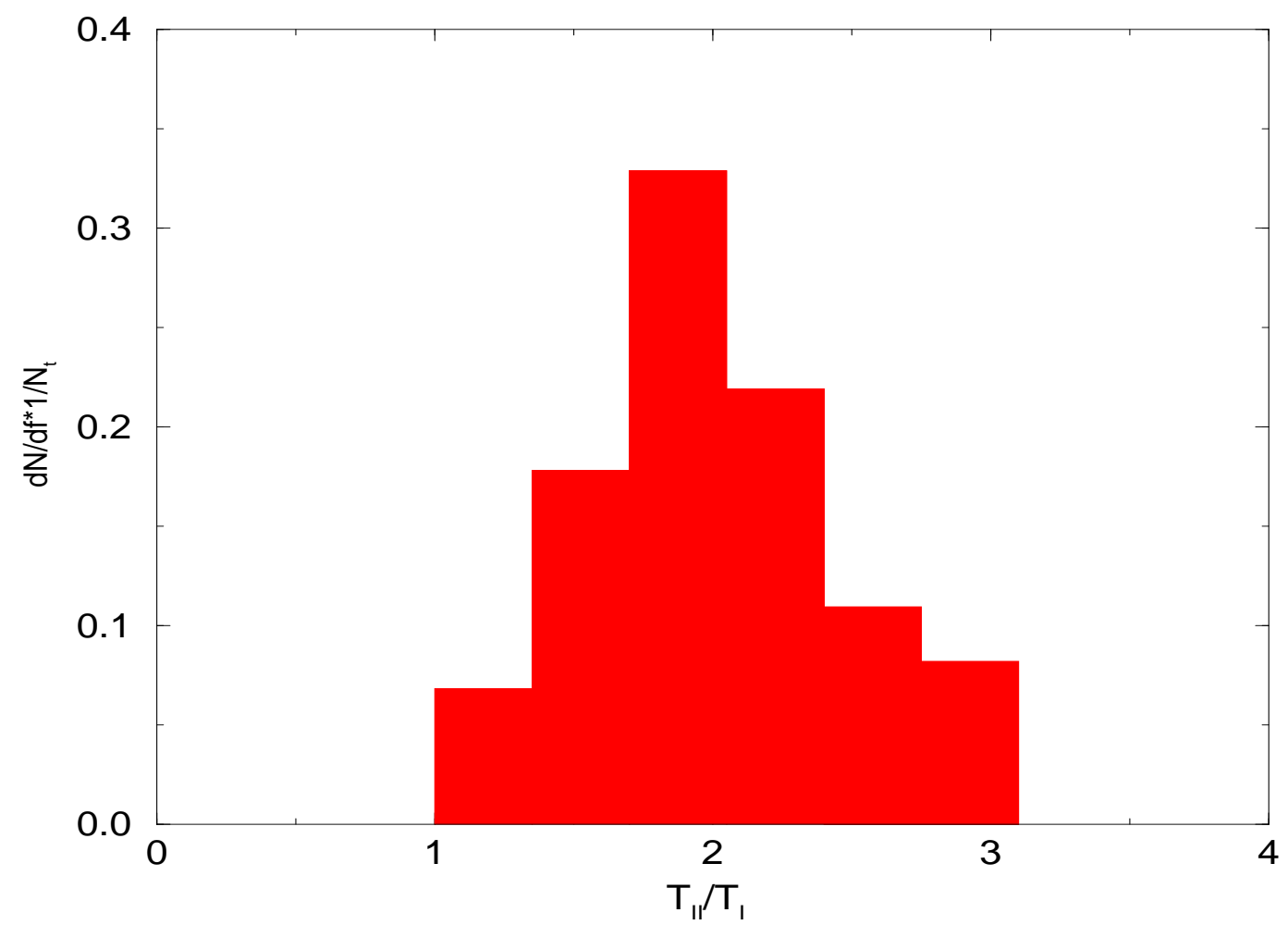

FIG. 4. Normalized distribution functions for the ratio of the frequencies of mode I and mode II clapping (sampling on 73 high-school students). 


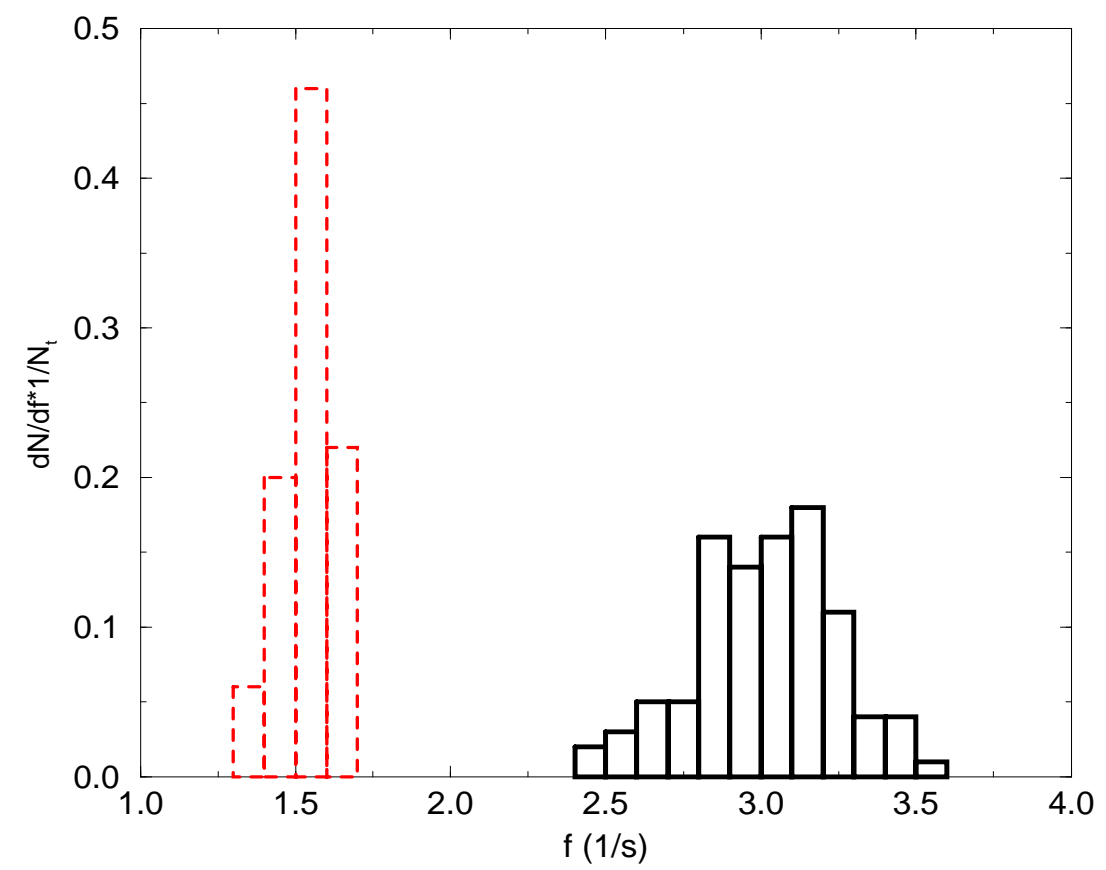

FIG. 5. Normalized distribution functions for the frequencies of mode I (continuous line) and mode II (dashed line) clapping of one chosen individual (sampling 100 times during a one week period).

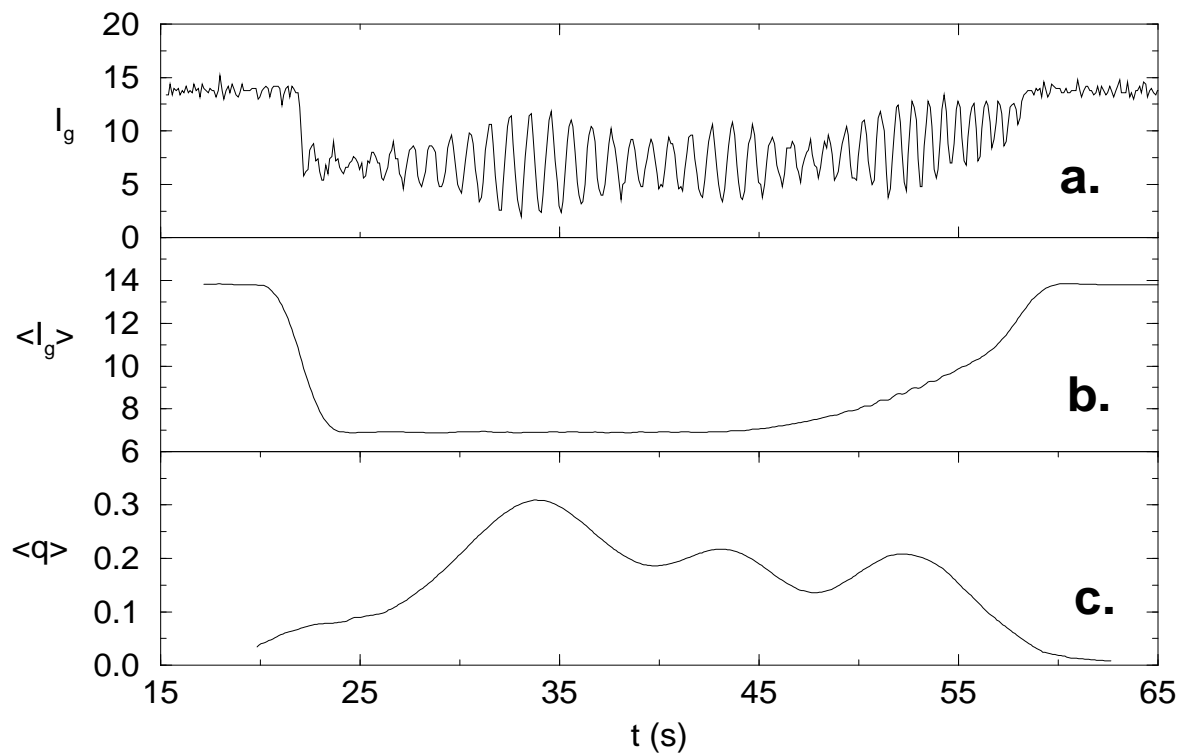

FIG. 6. Computer simulation of the Kuramoto model for $N=70$ rotators $\left(K=0.8 s^{-1}, \bar{\omega}=2 \pi s^{-1}\right.$ and $\left.D=2 \pi / 6.9 s^{-1}\right)$. We double the rotators periods at $t_{1}=21 \mathrm{~s}$ and linearly increase the frequency back to the original value from $t_{2}=35 \mathrm{~s}$. The noise-pulse given by one oscillator has $I_{0}=\omega / \bar{\omega}$ intensity and $\tau=0.01 \mathrm{~s}$ duration. 